\title{
Preparation of Monodisperse Polystyrenes with High Molecular Weights
}

\author{
Teruo Fujimoto and Mitsuru Nagasawa \\ Department of Synthetic Chemistry, Faculty of Engineering, \\ Nagoya University, Chikusa-ku, Nagoya, Japan.
}

(Received November 25, 1974)

\begin{abstract}
A polymerization procedure for polystyrenes having high molecular weight and sharp molecular weight distributions is reported. A drop of $n$-hexylbenzene solution of $n$-butylithium is added to a THF solution of styrene with strong stirring. The initiator can be uniformly distributed throughout the solution to react with impurities in the solution while the drop is dissolving into the THF. The molecular weight distribution of the polystyrenes thus prepared is much sharper than those of monodisperse polystyrenes available commercially and their molecular weights can be over 10 million.
\end{abstract}

KEY WORDS Monodisperse Polymer / Monodisperse Polystyrenes / High Molecular Weight Polystyrene / Anionic Polymerization / Living Polymerization /

The so-called monodisperse polystyrenes, which are prepared by the anionic polymerization technique, are now widely used in polymer science, for example, as standard polymers for polymer characterization or when studying the effect of polydispersity on physical properties of linear polymers. Various samples of monodisperse polystyrenes are now commercially available. However, the molecular weight distributions of those samples are not generally sharp enough and, moreover, the molecular weights of the samples available do not cover a high enough range of molecular weight. For example, to study the effect of molecular weight distribution on the viscoelastic properties of linear polymers, which are sometimes governed by an average molecular weight higher than the $z$-average or to use them as standard samples for GPC, the monodisperse polystrenes are required to have higher molcular weights and sharper molecular weight distributions than the samples available commercially at present. It is well known that polystyrenes polymerized by the anionic polymerization method have generally much broader molecular weight distribution than $\operatorname{poly}(\alpha$-methylstyrenes) which are also prepared by a similar anionic polymerization technique. ${ }^{1}$

The difference between the molecular weight distributions of polystyrene and poly ( $\alpha$-methylstyrene) arises from their differences in difficulty of purification and/or in polymerization rate: Although some reagents suitable for purification of styrene monomer are now found, ${ }^{2}$ the impurities in styrene monomer cannot perfectly be removed. Moreover, the polymerization rate of styrene is so high that the monomer has to be showered on the initiator solution by the method of Szwarc. $^{3}$ The main cause for the broad molecular weight distribution of polystyrenes may, therefore, be that some polystyryl anions are killed with the impurities during showering. On the other hand, $\alpha$-methylstyrene does not polymerize at room temperature and $\alpha$-methylstyrene monomer can therefore be mixed with an anionic initiator in the reaction vessel so that all the impurities may be reacted with the initiator before polymerization. ${ }^{1}$ Considering these differences between the properties of the two kinds of monomers, it can be immediately speculated that, if it is possible to react all the impurities in styrene with the initiator before the polymerization starts, the molecular weight distributions of polystyrenes obtained would be as sharp as those of $\operatorname{poly}(\alpha$-methylstyrenes). Some solution properties of polystyrenes having fairly high molecular weights and sharp molec- 
ular weight distributions were reported by Fukuda, et al., recently. ${ }^{4}$ It is noteworthy that an initiator solution was added to a mixture of styrene monomer and solvent in their preparation of samples, whereas monomer was added to a mixture of initiator and solvent in most previous experiments.

This is to report a preparation method of polystyrenes having extremely sharp molecular weight distributions based on the above idea. Using this method, moreover, it is possible to prepare samples having extremely high molecular weights since all impurities can be reacted with initiators before the polymerization starts. This polymerization method can be performed on a fairly large scale.

\section{EXPERIMENTAL PROCEDURES}

\section{Purification of Styrene Monomer and Solvents}

Commercial styrene monomer is purified by routine methods such as distillation with $\mathrm{LiAlH}_{4}$ in vacuo. The monomer thus purified is further distilled from a mixture of the monomer and triphenylmethyl sodium, which is prepared by reacting triphenylmethyl chloride with sodium metal in THF. ${ }^{5}$ Triphenylmethylsodium is kept as $0.2-M$ solution in diglyme. The polymerization of styrene monomer can hardly occur with the anion.

Tetrahydrofran (THF) is purified by distillation in vacuo in the presence of potassium $\alpha$ methylstyrene anion after the usual purification. Diglyme is also purified by a similar method.

\section{Preparation of Initiator}

The $n$-butyllithium is prepared in $n$-hexane by an ordinary method. ${ }^{1}$ The $n$-hexane is evaporated and $n$-butyllithium is dissolved in $n$-hexylbenzene. The concentration of $n$-butyllithium may be $0.1-0.3 N$. The $n$-hexylbenzene is chosen since it can be dissolved into THF at a proper speed, as explained later.

\section{Polymerization Procedure}

The apparatus as shown in Figure 1 is pumped to $10^{-6} \mathrm{mmHg}$ and sealed off. The whole apparatus is carefully washed with the cumylpotassium solution or the potassium $\alpha$-methylstyrene anion solution in flask $\mathrm{W}_{1}$, which can be recovered in flask $\mathrm{W}_{2}$. The anion remaining

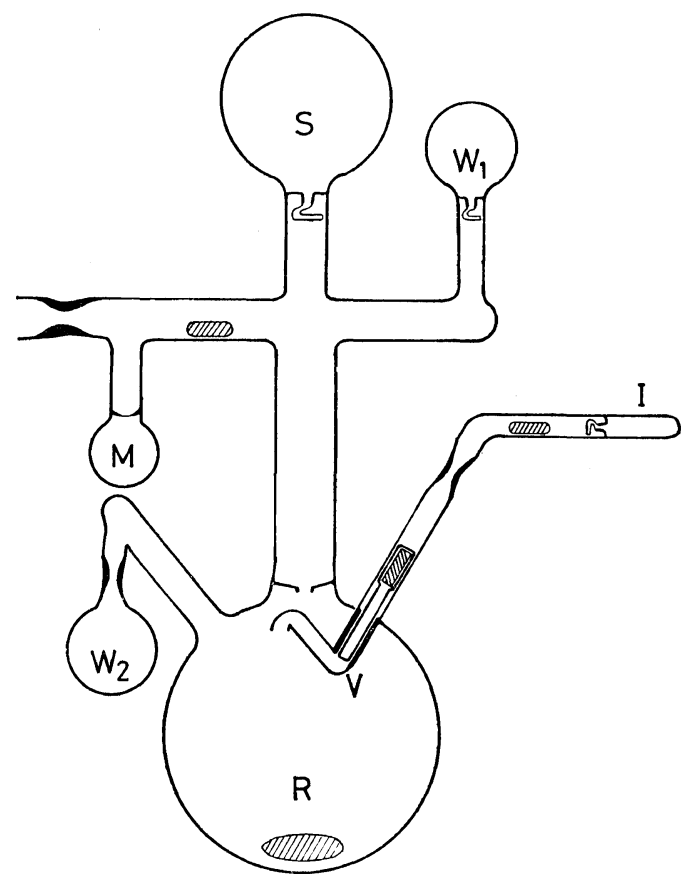

Figure 1. The polymerization apparatus.

on the glass wall is washed away with THF which is directly distilled from the THF solution of the washing reagent in $\mathrm{W}_{2}$. The flask $\mathrm{W}_{2}$ is sealed off. THF in flask $\mathrm{S}$ is introduced into the reaction vessel $\mathrm{R}$ and the styrene monomer in flask $M$ is transfered into the reaction vessel by distillation. The $n$-hexylbenzene solution of $n$-butyllithium in ampule $\mathrm{I}$ is transfered into $\mathrm{V}$ and it is then added dropwise to the reaction mixture by lowering a glass rod with a magnet. The initiators in the first drop may be killed by reaction with impurities in the reaction mixture. If no polymerization occurs with the first drop in about $20 \mathrm{~min}$, the second drop is added. The second drop of initiator usually causes the polymerization.

Since it takes time to dissolve $n$-hexylbenzene into THF at $-78^{\circ} \mathrm{C}$, all initiators are uniformly spread throughout the solution when the drop is dissolved. That is, the concentration of $n$ butyllithium in $n$-hexylbenzene and the size of the drop are controlled depending on the amount of polymerization mixture so that the drop may dissolve into the THF and spread the initiator throughout the solution uniformly. Usually it 
takes a few seconds and the whole impurity may be reacted with the initiator during the time.

In the present experiments, $20 \mathrm{~g}$ of styrene was polymerized in $4 l$ of THF.

\section{Fractionation}

The molecular weight distribution of polystyrene prepared by this method is very narrow without fractionation. However, the tail part of the polymer can be removed with no trouble when the polymer is precipitated in a mixture of methanol and toluene. The head part of the polymer is cut off by adding methanol to the toluene solution of the sample, to remove a fragment of the initiator used.

\section{Characterization Procedures}

The weight average molecular weights of the samples were determined by the light-scattering method in trans-decaline at $30^{\circ} \mathrm{C}$ using a Fica 50. The molecular weight distributions of the samples were checked by the sedimentationvelocity method in MEK at $25^{\circ} \mathrm{C}$ using an analytical ultracentrifuge Beckman-Spinco Model E. The sedimentation coefficient $S_{0}$ is related to the molecular weight of polystyrene by ${ }^{6}$

$$
S_{0}=5.3 \times 10^{-15} M_{w}^{0.44} \text { in MEK at } 25^{\circ} \mathrm{C}
$$

but this equation is applicable up to $M=3 \times 10^{6}$.

\section{RESULTS}

The weight average molecular weight of samples No. 201 and 401 were found to be $1.4 \times 10^{6}$ and $1.2 \times 10^{7}$, respectively. However, the figure for No. 401 is tentative because of its rather high value. If we calculate their molecular weights from their sedimentation coefficients using eq 1 , we have $M=1.8 \times 10^{6}$ and $1.8 \times 10^{7}$, respectively. The reason for the differences between both values is not clear but is not important for the present purpose.

Sedimentation patterns of the present samples and all other polystyrenes thus prepared show that their molecular weight distributions are very sharp in all cases. The distribution of sedimentation coefficient of No. 201 was read at different times and extrapolated to infinite time by the method of Baldwin and Williams to eliminate the effect of diffusion. ${ }^{7}$ The sedimentation constant distributions obtained at three polymer concentrations were extraporated to zero polymer

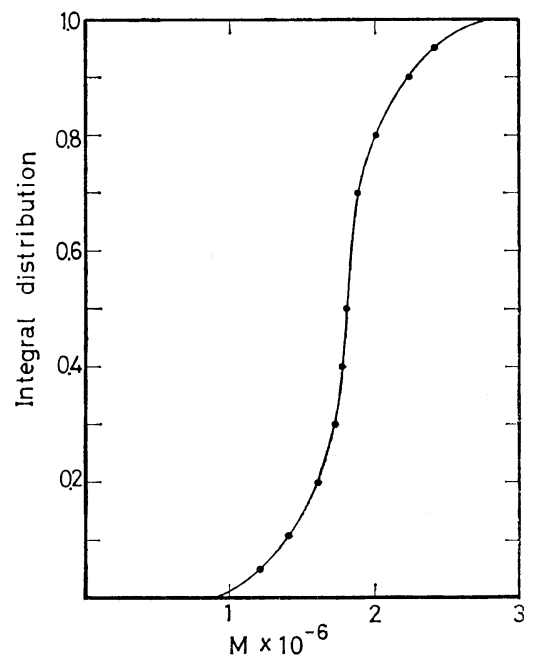

Figure 2. The integral molecular weight distribution curve of polystyrene No. 201 having $M_{w}=$ $1.4 \times 10^{6}$.

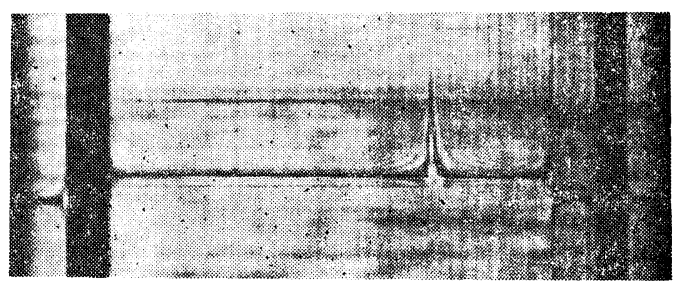

Figure 3. An example of sedimentation pattern of polystyrene No. 401 having $M_{w}=1.2 \times 10^{7}$ : solvent, MEK, concn, $0.0655 \mathrm{~g} / \mathrm{ml}$; speed of rotation, 25980 rpm; angle, $70^{\circ}$; time, $31 \mathrm{sec}$.

concentration. The final distribution curve of the sedimentation coefficient obtained can be converted to the molecular weight distribution of the sample if we assume that eq 1 is applicable. The molecular weight distribution curve of sample No. 201 thus determined is shown in Figure 2 as an example. The standard deviation of molecular weight from the numberaverage molecular weight may be estimated from the data in Figure 2 to be

$$
\frac{\Delta M}{M_{n}}=0.15
$$

The value of $M_{w} / M_{n}$ is related to the standard deviation by

$$
\frac{\Delta M}{M_{n}}=\left(\frac{M_{w}}{M_{n}}-1\right)^{1 / 2}
$$




\section{T. Fujimoto and M. NAgAsawa}

Thus, $M_{w} / M_{n}$ of No. 201 may be about 1.02 .

The molecular weight of another sample 401 was so high that it was difficult to determine the sedimentation coefficient distribution of this sample by the sedimentation-velocity method. A sedimentation pattern obtained for a solution of concentration $0.0655 \mathrm{~g} / \mathrm{d} l$ at $25980 \mathrm{rpm}$ are shown in Figure 3 to show that the molecular weight distribution of the sample is very sharp. The sedimentation pattern does not significantly diffuse with time even at low concentrations.

\section{DISCUSSION}

The polymerization rate of the free polystyryl anion is so much faster than that of its ion-pair and the initiator concentration in the present experiments is so low that it may be assumed that the propagation rate of the polystyryl anion is proportional to the product of the concentration of free styryl anion [LE] $(\mathrm{mol} / \mathrm{l})$ and that of the styrene monomer $[\mathrm{M}](\mathrm{g} / l)$ :

$$
-\mathrm{d}[\mathrm{M}] / \mathrm{d} t=K[\mathrm{LE}][\mathrm{M}]
$$

where $k$ is the propagation rate constant of the free polystyryl anion. The molecular weight of polystyrenes obtained, $M_{n}$, is given by the ratio of the amount of styrene polymerized and the concentration of active end such as

$$
\begin{aligned}
M_{n} & =\left([\mathrm{M}]_{0}-[\mathrm{M}]\right) /[\mathrm{LE}] \\
& =[\mathrm{M}]_{0} \cdot x /[\mathrm{LE}]
\end{aligned}
$$

where $[M]_{0}$ is the initial concentration of monomer $(\mathrm{g} / l)$ and $x$ is the conversion at time $t$. By integrating eq 2 and using the relationship of eq 3 , we have

$$
M_{n}=\frac{[\mathrm{M}]_{0}}{[\mathrm{LE}]}\left(1-\mathrm{e}^{-k[\mathrm{LE}] t}\right)
$$

Since the molecular weight distribution arising from the statistical reason in propagation is almost negligible, ${ }^{8}$ it may be assumed that the molecular weight distributions of the present samples arise mainly from the finite time required to mix the initiator with monomer. If $\Delta t$ is required for the mixing, the polystyrene obtained may have an ambiguity in its molecular weight by

$$
\Delta M_{n}=\frac{[\mathrm{M}]_{0}}{[\mathrm{LE}]}\left[1-\mathrm{e}^{-k[\mathrm{LE}] \Delta t / 2}\right]
$$

Thus, the dispersion of molecular weight in the polystyrene may be expressed by

$$
\frac{\Delta M_{n}}{M_{n}}=\frac{\left[1-\mathrm{e}^{-k[\mathrm{LE}] \Delta t / 2}\right]}{\left[1-\mathrm{e}^{-k[\mathrm{LE}] t}\right]}
$$

In most experiments, we have

$$
\left[1-\mathrm{e}^{-k[\mathrm{LE}] t}\right] \doteqdot 1
$$

and, hence,

$$
\frac{\Delta M_{n}}{M_{n}} \doteqdot\left[1-\mathrm{e}^{-k[\mathrm{LE}] \Delta t / 2}\right]
$$

In the present experiments, [LE] can be calculated from the amounts of polymer obtained and their molecular weights: [LE] $=3.6 \times 10^{-6}$ and $4.2 \times 10^{-7} \mathrm{~mol} / l$ for experiment No. 201 and 401, respectively. If we estimate $\Delta t$ by adding a drop of ink into water at the same experimental conditions but at room temperature, $\Delta t$ is about $2-3 \mathrm{sec}$. Since the initiator drop is dissolved into THF at $-78^{\circ} \mathrm{C}$, which has a little higher viscosity than water, $\Delta t$ in the present experiments may be a little longer than $2-3 \mathrm{sec}$. Let us assume $\Delta t=6 \mathrm{sec}$ here.

The anionic polymerization kinetics of styrene were extensively studied by Szwarc and his coworkers. ${ }^{9}$ The propagation rate constant of free styryl anion with $\mathrm{Li}^{+}$counter ion in $\mathrm{THF}$, however, has not yet been determined. The rate constant with $\mathrm{Na}^{+}$and $\mathrm{Cs}^{+}$counter ions in THF were determined at various temperatures, while the value with $\mathrm{Li}^{+}$was determined in tetrahydropyrane (THP). It appears to be reasonable from those data to assume that the propagation rate constant of free anion is almost independent of counter ion species and solvent. The propagation constant of free polystyryl anion in THF at $-78^{\circ} \mathrm{C}$ estimated from the above data is $3-10$ $\times 10^{3}(l / \mathrm{mol} \mathrm{sec})$.

Using the propagation constant $k$, [LE] and $\Delta t$ thus estimated, we can calculate $\Delta M_{n} / M_{n}$ from eq 7 , such as

$$
\begin{aligned}
\Delta M_{n} / M_{n} & =0.11-0.03 \text { for No. } 201 \\
& =0.013-0.004 \text { for No. } 401
\end{aligned}
$$

It is reasonable that sample Nos. 401 and 201 have extremely sharp molecular weight distributions. However, the molecular weight distribution of sample No. 201 observed is still broader 
than the distribution thus estimated. If we choose a more proper experimental condition, the molecular weight distribution could be sharper.

As can be seen from eq 7, the value of $\Delta M / M$ is governed by $\Delta t$ if $[M]_{0}$ and [LE] are kept constant. To keep the standard deviation of molecular weight $\Delta M / M$ within $10 \%$, that is, to obtain $M_{w} / M_{n} \leq 1.01$ for the samples having $M_{n}=1.4 \times 10^{6}$ and $1.2 \times 10^{7}, \Delta t$ should be shorter than 6 and $48 \mathrm{sec}$, respectively. This time for mixing the initiator with monomers uniformly may determine the upper limit of the polymerization scale by this method, though the polymerization condition should still be improved.

Acknowledgment. The light-scattering experiments were carried out by Mr. T. Shima and the molecular weight distributions were determined by Mr. H. Kajiura and M. Ushiyama. The authors wish to thank them for their helpful cooperation and also Mr. T. Watanabe for designing the polymerization apparatus.

\section{REFERENCES}

1. T. Fujimoto, N. Ozaki, and M. Nagasawa, $J$. Polym. Sci., Part-A, 3, 2259 (1965).

2. F. Wenger and S. P.S. Yen, Makromol. Chem., 43, 1 (1961); D. McIntyre, L. J. Fetters, and E. Slagowski, Science, 176, 1041 (1972).

3. M. Szwarc, M. Levy, and R. McKovitch, J. Amer. Chem. Soc., 78, 2656 (1956).

4. M. Fukuda, M. Fukutomi, T. Kato, and T. Hashimoto, J. Polym. Sci., 12, 871 (1974).

5. T. Fukutomi, private communication.

6. K. Kawahara, Makromol. Chem., 73, 1 (1964).

7. H. Fujita, "Mathematical Theory of Seddimentation Analysis", Academic Press Inc., New York, N. Y., 1962.

8. W. B. Brown and M. Szwarc, Trans. Faraday Soc., 54, 416 (1958).

9. T. Shimomura, K. J. Tölle, J. Smid, and M. Szwarc, J. Amer. Chem. Soc., 89, 796 (1967). 